\title{
ON THE IDEAL STRUCTURE OF ALGEBRAS OF STAR-ALGEBRA VALUED FUNCTIONS
}

\author{
JORMA ARHIPPAINEN
}

(Communicated by Palle E. T. Jorgensen)

\begin{abstract}
The ideal structure of the algebra $C(X, A)$ has been studied in many papers under various topological assumptions on the space $X$ and the algebra $A$. In this paper we shall study the case where $X$ is a completely regular topological space and $A$ is a locally convex star algebra. In such case the structure of closed (proper) ideals can be described not only by using points of $X$ and some family of closed ideals of $A$, as usual, but also by using points of the carrier space $\Delta(A)$ of $A$ and some family of closed ideals of $C(X, A)$ depending on those points and also by using different kind of slice ideals of $C(X, A)$.
\end{abstract}

\section{INTRODUCTION}

Let $A$ be a commutative locally $m$-convex algebra with identidy $e$ over the field $C$ of the complex numbers. Let $\mathscr{P}=\left\{p_{\lambda} \mid \lambda \in \Lambda\right\}$ be a family of seminorms which defines the topology in $A$ denoted by $T(\mathscr{P})$. It is assumed that $T(\mathscr{P})$ is a Hausdorff topology; in other words, $p_{\lambda}(x)=0$ for all $\lambda \in \Lambda$ only, if $x=0$. Furthermore, we assume that the family $\mathscr{P}$ is directed. For $\lambda \in \Lambda$ we shall denote $N_{\lambda}=\left\{x \in A \mid p_{\lambda}(x)=0\right\}$. For each $\lambda \in \Lambda$ the quotient algebra $A / N_{\lambda}=A_{\lambda}$ is a normed algebra with a norm $\dot{p}_{\lambda}\left(x+N_{\lambda}\right)=p_{\lambda}(x)$, $x+N_{\lambda} \in A / N_{\lambda}$.

Let $\Delta(A)$ be the set of all non-trivial continuous $C$-homomorphisms on $A$. The set $\Delta(A)$ will be equipped with the relative $\sigma\left(A^{\prime}, A\right)$-topology called the Gelfand topology. With this topology $\Delta(A)$ is called the carrier space of $(A, T(\mathscr{P}))$.

Let $x \in A$ be given. If we define a $C$-valued function $\hat{x}$ on the carrier space $\Delta(A)$ by $\widehat{x}(\tau)=\tau(x), \tau \in \Delta(A)$, then $\widehat{x}$ is continuous, whence $\widehat{x} \in C(\Delta(A))$.

Let $I$ be an ideal of $A$. The hull of $I$ denoted by $h(I)$ is then defined as $h(I)=\{\tau \in \Delta(A) \mid \widehat{x}(\tau)=0, x \in I\}$. The kernel $k(E)$ of a subset $E$ of $\Delta(A)$ is defined by $k(E)=\{x \in A \mid \widehat{x}(\tau)=0, \tau \in E\}$, and for an empty set $\varnothing$ we define $k(\varnothing)=A$. Obviously $h(I)$ is a closed subset of $\Delta(A)$ and $k(E)$ is a closed ideal of $(A, T(\mathscr{P}))$.

Received by the editors December 7, 1992 and, in revised form, April 26, 1993.

1991 Mathematics Subject Classification. Primary 46J20, 46J25, 46K10. 
For a completely regular topological space $X$ denote by $C(X, A)$ the set of all continuous $A$-valued functions defined on $X$. Algebraic operations in $C(X, A)$ are defined pointwise.

Let $\mathscr{K}$ be the compact cover of $X$ which is closed under finite unions, in other words, $\mathscr{K}$ is a family of subsets of $X$ for which each $K \in \mathscr{K}$ is compact and

$$
\cup\{K \mid K \in \mathscr{K}\}=X,
$$

if $K_{1}$ and $K_{2} \in \mathscr{K}$, then $K_{1} \cup K_{2} \in \mathscr{K}$.

For $K \in \mathscr{K}$ and $\lambda \in \Lambda$, we define a seminorm $p_{(K, \lambda)}$ on $C(X, A)$ by

$$
p_{(K, \lambda)}(f)=\sup _{t \in K} p_{\lambda}(f(t)), \quad f \in C(X, A) .
$$

We write $\mathscr{P}(\mathscr{K}, \Lambda)=\left\{p_{(K, \lambda)} \mid K \in \mathscr{K}, \lambda \in \Lambda\right\}$. The family $\mathscr{P}(\mathscr{K}, \Lambda)$ defines a locally $m$-convex Hausdorff topology on $C(X, A)$ denoted from now on by $T(\mathscr{K}, \Lambda)$. Obviously $\mathscr{P}(\mathscr{K}, \Lambda)$ is a directed family of seminorms. If $\mathscr{K}=\mathscr{K}(X)=$ the set of all compact subsets of $X$, then $T(\mathscr{K}, \Lambda)$ is the compact-open topology of $C(X, A)$. Given $K \in \mathscr{K}$ and $\lambda \in \Lambda$, denote by $N_{(K, \lambda)}=\left\{f \in C(X, A) \mid p_{(K, \lambda)}(f)=0\right\}$.

Let $t \in X$, and let $I$ be an ideal of $A$. We define an ideal $J_{(t, I)}$ of $C(X, A)$ by $J_{(t, I)}=\{f \in C(X, A) \mid f(t) \in I\}$ and furthermore $C(X, I)=\{f \in$ $C(X, A) \mid f(t) \in I, t \in X\}$. Obviously $C(X, I)=\cap_{t \in X} J_{(t, I)}$.

For a given subset $E \subset X(E \neq \varnothing)$ we denote by $J(E, A)=\{f \in$ $C(X, A) \mid f(t)=0, t \in E\}$. It is easy to see that $J(E, A)$ is a closed ideal of $(C(X, A), T(\mathscr{K}, \Lambda))$, if $E$ is a closed subset of $X$. Furthermore, if $I$ is a closed ideal of $(A, T(\mathscr{P}))$ and $t$ is a given point of $X$, then both of the ideals $J_{(t, I)}$ and $C(X, I)$ are closed in $(C(X, A), T(\mathscr{K}, \Lambda))$.

Let $K \in \mathscr{K}, \lambda \in \Lambda$, and $\epsilon>0$. We denote by $V_{(K, \lambda)}(\epsilon)$ the set $V_{(K, \lambda)}(\epsilon)=$ $\left\{f \in C(X, A) \mid p_{(K, \lambda)}(f)<\epsilon\right\}$. Obviously the sets $V_{(K, \lambda)}(\epsilon), K \in \mathscr{K}, \lambda \in \Lambda$, and $\epsilon>0$ form a subbase of neighbourhoods of zero. (See [17], p. 8.)

\section{ON FUNCTIONAL REPRESENTATION OF $(C(X, A), T(\mathscr{K}, \Lambda))$}

We shall first study some properties of the carrier space $\Delta(A)$. One of the basic results is that $\Delta(A)$ can be expressed as the union of certain compact subsets of it. Namely, it has been shown in [15] that $\Delta(A)=\cup_{\lambda \in \Lambda} K_{\lambda}$ where $K_{\lambda}=$ the polar of $V_{\lambda}(1) \cap \Delta(A)$. We shall now express this property of $\Delta(A)$ by using hulls of the nullideals $N_{\lambda}, \lambda \in \Lambda$.

Lemma 1. Let $(A, T(\mathscr{P}))$ be a commutative locally $m$-convex topological algebra. Then $\left\{h\left(N_{\lambda}\right) \mid \lambda \in \Lambda\right\}$ is a compact cover of $\Delta(A)$ which is closed under finite unions. Furthermore, if $\tau \in h\left(N_{\lambda}\right)$, then $|\tau(x)| \leq p_{\lambda}(x)$ for all $x \in A$.

Proof. Let $\tau \in \Delta(A)$ be arbitrary. Since $\tau$ is continuous, there is $\lambda \in \Lambda$ and a real number $M>0$ such that $|\tau(x)| \leq M p_{\lambda}(x)$ for all $x \in A$. Thus, if $x \in N_{\lambda}$, then $|\tau(x)| \leq M p_{\lambda}(x)=0$ which implies that $\widehat{x}(\tau)=\tau(x)=0$ for all $x \in N_{\lambda}$ and we can see that $\tau \in h\left(N_{\lambda}\right)$. So $\Delta(A)=\cup_{\lambda \in \Lambda} h\left(N_{\lambda}\right)$. Let $\lambda \in \Lambda$ be arbitrary. By Theorem 4.1 and Corollary 2.1 of [14] $h\left(N_{\lambda}\right)$ is homeomorphic to $\Delta\left(\tilde{A_{\lambda}}\right)$. Since $\Delta\left(\widetilde{A_{\lambda}}\right)$ is compact, we can see that $h\left(N_{\lambda}\right)$ is compact for each $\lambda \in \Lambda$. If $\lambda_{1}$ and $\lambda_{2} \in \Lambda$, then there is $\lambda_{M} \in \Lambda$ such that $\max \left\{p_{\lambda_{1}}, p_{\lambda_{2}}\right\}=p_{\lambda_{M}}$ (since we assumed that $\mathscr{P}$ is a directed family of seminorms on $A$ ). Now 
$h\left(N_{\lambda_{1}}\right) \cup h\left(N_{\lambda_{2}}\right)=h\left(N_{\lambda_{1}} \cap N_{\lambda 2}\right)=h\left(N_{\lambda_{M}}\right)$ which shows that the family $\left\{h\left(N_{\lambda}\right) \mid\right.$ $\lambda \in \Lambda\}$ is closed under finite unions.

If $\tau \in h\left(N_{\lambda}\right)$, then we can define a complex homomorphism $\tau_{\lambda}$ on $A_{\lambda}$ by $\tau_{\lambda}\left(x+N_{\lambda}\right)=\tau(x), x+N_{\lambda} \in A_{\lambda}$. It is easy to see that $\tau_{\lambda}$ is well defined. Now $A_{\lambda}$ is a normed algebra with a norm $\dot{p}_{\lambda}$. Let $\tilde{A}_{\lambda}$ be the completion of $A_{\lambda}$ and denote by $\widetilde{\dot{p}}_{\lambda}$ the corresponding extended norm on $\widetilde{A}_{\lambda}$. Now we can consider $A_{\lambda}$ as a dense subalgebra of $\widetilde{A}_{\lambda}$ and we have $\widetilde{\dot{p}}_{\lambda}\left(x+N_{\lambda}\right)=\dot{p}_{\lambda}\left(x+N_{\lambda}\right)$ for all $x+N_{\lambda} \in A_{\lambda}$. Denote by $\tilde{\tau}_{\lambda}$ the extension of $\tau_{\lambda}$ to $\tilde{A}_{\lambda}$. The mapping $\tau_{\lambda} \mapsto$ $\tilde{\tau}_{\lambda}, \tau_{\lambda} \in \Delta\left(A_{\lambda}\right)$, is a homeomorphism from $\Delta\left(A_{\lambda}\right)$ onto $\Delta\left(\tilde{A}_{\lambda}\right)$ and obviously we have $\tilde{\tau}_{\lambda}\left(x+N_{\lambda}\right)=\tau_{\lambda}\left(x+N_{\lambda}\right)$ for all $x+N_{\lambda} \in A_{\lambda}$. Since $\widetilde{A}_{\lambda}$ is a commutative Banach algebra, we naturally have $\left|\tilde{\tau}_{\lambda}\left(\widetilde{x}_{\lambda}\right)\right| \leq \widetilde{\dot{p}}_{\lambda}\left(\widetilde{x}_{\lambda}\right)$ for all $\widetilde{x}_{\lambda} \in \tilde{A}_{\lambda}$. Thus we obtain

$$
|\tau(x)|=\left|\tau_{\lambda}\left(x+N_{\lambda}\right)\right|=\left|\tilde{\tau}_{\lambda}\left(x+N_{\lambda}\right)\right| \leq \tilde{\dot{p}}_{\lambda}\left(x+N_{\lambda}\right)=\dot{p}_{\lambda}\left(x+N_{\lambda}\right)=p_{\lambda}(x)
$$

for all $x \in A$.

For a given $x \in A$ we shall define $p_{h\left(N_{\lambda}\right)}(x)=\sup _{\tau \in h\left(N_{\lambda}\right)}|\widehat{x}(\tau)|$. By Lemma 1 we have $|\hat{x}(\tau)| \leq p_{\lambda}(x)$ for all $x \in A$ and $\tau \in h\left(N_{\lambda}\right)$. Thus we have $p_{h\left(N_{\lambda}\right)}(\widehat{x}) \leq p_{\lambda}(x)$ for all $x \in A$ and $\lambda \in \Lambda$. The family $\left\{p_{h\left(N_{\lambda}\right)} \mid \lambda \in \Lambda\right\}$ of seminorms defines a locally m-convex topology on $\widehat{A}$. Denote this topology by $T(\Lambda)$. Since $p_{h\left(N_{\lambda}\right)}(\widehat{x}) \leq p_{\lambda}(x)$ for all $x \in A$ and $\lambda \in \Lambda$, we can see that the Gelfand mapping $x \mapsto \widehat{x}, x \in A$ from $(A, T(\mathscr{P}))$ onto $(\widehat{A}, T(\Lambda))$ is continuous.

Let $(A, T(\mathscr{P}))$ be a locally $m$-convex algebra. If $p_{\lambda}\left(x^{2}\right)=p_{\lambda}(x)^{2}$, for all $x \in A$ and $\lambda \in \Lambda$, we shall say that $(A, T(\mathscr{P}))$ is a square algebra.

Remark. If $(A, T(\mathscr{P}))$ is a commutative locally convex square algebra, then it is automatically $m$-convex. (See [6].)

If $(A, T(\mathscr{P}))$ is a square algebra, then we have $p_{\lambda}(x)=p_{h\left(N_{\lambda}\right)}(\widehat{x})$ for all $x \in A$ and $\lambda \in \Lambda$. This means that we can identify $(A, T(\mathscr{P}))$ and $(\widehat{A}, T(\Lambda))$ as locally $m$-convex algebras.

Now the following lemmas are easy to verify, whence the proofs will be omitted.

Lemma 2. If $(A, T(\mathscr{P}))$ is a square algebra, then the carrier spaces $\Delta(A)$ and $\Delta(\widehat{A})$ are homeomorphic.

Lemma 3. $(C(X, A), T(\mathscr{K}, \Lambda))$ is a square algebra if and only if $(A, T(\mathscr{P}))$ is a square algebra.

If $(A, T(\mathscr{P}))$ is a square algebra, then by [4] Corollary 3.1 we have $k\left(h\left(N_{\lambda}\right)\right)$ $=N_{\lambda}$ for all $\lambda \in \Lambda$. By using this fact and Lemmas 1 and 2 of [5] we can obtain the following results.

Theorem 1. Suppose that $(A, T(\mathscr{P}))$ is a square algebra. Then

$1^{\circ} \cdot k\left(h\left(N_{(K, \lambda)}\right)\right)=N_{(K, \lambda)}, K \in \mathscr{K}$ and $\lambda \in \Lambda$,

$2^{\circ}$. $k\left(h\left(J_{\left(t, N_{\lambda}\right)}\right)\right)=J_{\left(t, N_{\lambda}\right)}, t \in X$ and $\lambda \in \Lambda$,

$3^{\circ}$. $\cap_{\tau \in h\left(N_{\lambda}\right)} C(X, \operatorname{ker} \tau)=C\left(X, N_{\lambda}\right), \lambda \in \Lambda$.

We shall say that $(A, T(\mathscr{P}))$ is a star algebra, if there is an involution $x \mapsto x^{*}, x \in A$, in $A$ such that $p_{\lambda}\left(x x^{*}\right)=p_{\lambda}(x)^{2}, x \in A, \lambda \in \Lambda$. The 
properties of star algebras can be found for example in [4], [7], [15], and [16]. See also [2].

If there is an involution in $A$, then we can define an involution $f \mapsto f^{*}$, $f \in C(X, A)$, in $C(X, A)$ by $f^{*}(t)=f(t)^{*}, f \in C(X, A), t \in X$.

It is easy to prove that the following result is valid.

Lemma 4. $(C(X, A), T(\mathscr{K}, \Lambda))$ is a star algebra, if and only if $(A, T(\mathscr{P}))$ is a star algebra.

We shall say that $(A, T(\mathscr{P}))$ has a property of spectral synthesis if $k(h(I))=$ $I$ for each closed ideal $I$ of $A$.

Theorem 2. Suppose that $(A, T(\mathscr{P}))$ is a star algebra for which $\widehat{A}=C(\Delta(A))$; then $(C(X, A), T(\mathscr{K}, \Lambda))$ has a property of spectral synthesis.

Proof. This result follows from Corollary 11 of [5] and Theorem 4.2 of [4].

Next we shall consider the Gelfand function of the algebra $(C(X, A)$, $T(\mathscr{K}, \Lambda))$. By [5] each $\phi \in \Delta(C(X, A))$ is of the form $\phi=\phi_{(t, \tau)}$ for some $(t, \tau) \in X \times \Delta(A)$, where $\phi_{(t, \tau)}(f)=\tau(f(t)), f \in C(X, A)$. If we define a mapping $\varphi: X \times \Delta(A) \mapsto \Delta(C(X, A))$ by $\varphi(t, \tau)=\phi_{(t, \tau)},(t, \tau) \in X \times \Delta(A)$, then by Theorem 5 of [5] $\varphi$ is a bijection for which the inverse mapping $\varphi^{-1}$ is continuous. Furthermore, if $\Delta(A)$ is locally equicontinuous, then $\varphi$ is continuous. Thus for a locally equicontinuous $\Delta(A)$ the product space $X \times \Delta(A)$ and the carrier space $\Delta(C(X, A))$ are homeomorphic.

Now

$$
\widehat{f}(\phi)=\widehat{f}\left(\phi_{(t, \tau)}\right)=\phi_{(t, \tau)}(f)=\tau(f(t)),
$$

and therefore we can consider the function $\widehat{f}$ also as a function on $X \times \Delta(A)$.

From now on we shall denote

$$
\widehat{f}(t, \tau)=\widehat{f}\left(\phi_{(t, \tau)}\right)=\tau(f(t)), \quad f \in C(X, A),(t, \tau) \in X \times \Delta(A) .
$$

Obviously $\widehat{f} \in C(X \times \Delta(A))$, if $\Delta(A)$ is locally equicontinuous (see [13] ).

Let $Y$ be a completely regular Hausdorff space and let $\mathscr{K}$ be a compact cover of $Y$ which is closed under finite unions. If we define a family $\left\{p_{K} \mid K \in \mathscr{K}\right\}$ of seminorms on $C(Y)$ by

$$
p_{K}(g)=\sup _{t \in K}|g(t)|, \quad g \in C(Y), K \in \mathscr{K},
$$

and denote the topology of $C(Y)$ generated by these seminorms by $T(\mathscr{K})$, then $(C(Y), T(\mathscr{K}))$ is a locally $m$-convex algebra with many interesting properties.

For example $\Delta(C(Y))=\left\{\tau_{t} \mid t \in Y\right\}$ where the mapping $\tau_{t}: C(Y) \rightarrow C$ is defined by

$$
\tau_{t}(g)=g(t), \quad g \in C(Y),
$$

moreover the mapping $t \mapsto \tau_{t}, t \in Y$, is a homeomorphism from $Y$ onto $\Delta(C(Y))$. (See [15], Example 7.6.) Furthermore, each closed ideal $I$ of $(C(Y)$, $T(\mathscr{K}))$ is of the form

$$
I=\{g \in C(Y) \mid g(t)=0, t \in E\}
$$


for some closed subset $E \subset Y$. ( See [4], Lemma 2.1, part (ii).) Since $\Delta(C(Y)$ ) and $Y$ can be identified as topological spaces, we shall from now on define the kernel operation on $\Delta(C(Y))=Y$ by

$$
k(E)=\{g \in C(Y) \mid g(t)=0, t \in E\}, \quad \text { when } E \subset Y \text { and } E \neq \varnothing,
$$

and for an empty set we define $k(\varnothing)=C(Y)$. Correspondingly we shall define the hull operation on $C(Y)$ by

$$
h(I)=\{t \in Y \mid g(t)=0, g \in I\},
$$

if $I$ is an ideal of $C(Y)$.

If $(A, T(\mathscr{P}))$ is a complete star algebra, then $\widehat{A}=C(\Delta(A))$ ( such an algebra is called full in [15]). For a proof see [4] or [15]. If we take $(A, T(\mathscr{P}))=$ $(C(Y), T(\mathscr{K}))$, then it is easy to see that $\hat{A}=C(\Delta(A))$ without an asumption that $(C(Y), T(\mathscr{K}))$ is complete. Thus the completeness is not a necessary condition for $A$ to be full.

Lemma 5. If $(A, T(\mathscr{P}))$ is a square algebra, then

$$
p_{(K, \lambda)}(f)=p_{h\left(N_{(K, \lambda)}\right)}(\widehat{f})=p_{K \times h\left(N_{\lambda}\right)}(\widehat{f}), \quad f \in C(X, A), K \in \mathscr{K}, \lambda \in \Lambda,
$$

where

$$
p_{h\left(N_{(K, \lambda)}\right)}(\widehat{f})=\sup _{\phi \in h\left(N_{(K, \lambda)}\right)}|\widehat{f}(\phi)|, \quad f \in C(X, A),
$$

and

$$
p_{K \times h\left(N_{\lambda}\right)}(\widehat{f})=\sup _{(t, \tau) \in K \times h\left(N_{\lambda}\right)}|\widehat{f}(t, \tau)|, \quad f \in C(X, A) .
$$

Proof. By Theorem 1 and Lemma 1.4 of [4] $(A, T(\mathscr{P}))$ is a square algebra, if and only if $p_{\lambda}(x)=p_{h\left(N_{\lambda}\right)}(\widehat{x})$ for all $x \in A$ and $\lambda \in \Lambda$. Thus our result follows from this fact and Corollary 10 of [5].

By Lemma 1 the family $\left\{K \times h\left(N_{\lambda}\right) \mid K \in \mathscr{K}, \lambda \in \Lambda\right\}$ is a compact cover of the product space $X \times \Delta(A)$ which is closed under finite unions. Thus, if we define the family $\left\{p_{K \times h\left(N_{\lambda}\right)} \mid K \in \mathscr{K}, \lambda \in \Lambda\right\}$ of seminorms on $C(X \times \Delta(A))$, then this family defines a locally $m$-convex topology on $C(X \times \Delta(A))$ denoted from now on by $T(\mathscr{K} \times \Lambda)$.

Now, if $\Delta(A)$ is locally equicontinuous, then $\widehat{f} \in C(X \times \Delta(A))$ for each $f \in C(X, A)$, whence $C(X, A)^{\wedge}=\{\widehat{f} \mid f \in C(X, A)\} \subset C(X \times \Delta(A))$.

Lemma 6. If $\Delta(A)$ is locally equicontinuous, then the mapping $f \mapsto \widehat{f}, f \in$ $C(X, A)$, from $(C(X, A), T(\mathscr{K}, \Lambda))$ into $(C(X \times \Delta(A)), T(\mathscr{K} \times \Lambda))$ is continuous.

Proof. We have $|\phi(f)| \leq p_{(K, \lambda)}(f)$ for all $f \in C(X, A), \phi \in h\left(N_{(K, \lambda)}\right)$, $K \in \mathscr{K}$, and $\lambda \in \Lambda$. Therefore

$$
p_{K \times h\left(N_{\lambda}\right)}(\widehat{f})=\sup _{(t, \tau) \in K \times h\left(N_{\lambda}\right)}|\widehat{f}(t, \tau)|=\sup _{\phi \in h\left(N_{(K, \lambda)}\right)}|\widehat{f}(\phi)| \leq p_{(K, \lambda)}(f)
$$

for all $f \in C(X, A), K \in \mathscr{K}, \lambda \in \Lambda$, from which the result follows. 
Next we shall give sufficient conditions under which we have

$$
C(X, A)^{\wedge}=C(X \times \Delta(A))
$$

and

$$
p_{(K, \lambda)}(f)=p_{K \times h\left(N_{\lambda}\right)}(\widehat{f}) \quad \text { for all } f \in C(X, A), K \in \mathscr{K}, \lambda \in \Lambda .
$$

If condition (4) is valid, then the algebras $(C(X, A), T(\mathscr{K}, \Lambda))$ and $\left(C(X, A)^{\wedge}, T(\mathscr{K} \times \Lambda)\right)$ are semi-isometrically isomorphic in the sense of [4]. Thus, if conditions (3) and (4) above are fulfilled, then $(C(X, A), T(\mathscr{K}, \Lambda))$ and $(C(X \times \Delta(A)), T(\mathscr{K} \times \Lambda))$ can be identified as topological algebras, which helps us to describe in this particular case many properties of the algebra $(C(X, A), T(\mathscr{K}, \Lambda))$.

Theorem 3. If $(A, T(\mathscr{P}))$ is a star algebra for which $\hat{A}=C(\Delta(A))$, then the mapping $f \mapsto \hat{f}, f \in C(X, A)$, has both of the properties (3) and (4).

Proof. Each star algebra is also a square algebra. (See [7], p. 261.) Thus condition (4) is valid by Lemma 2 . So it suffices to prove condition (3). Let $g \in C(X \times \Delta(A))$ be arbitrary. Now for each fixed $t_{0} \in X$ the function $g_{t_{0}}$ defined by

$$
g_{t_{0}}(\tau)=g\left(t_{0}, \tau\right), \quad \tau \in \Delta(A),
$$

belongs to $C(\Delta(A))=\widehat{A}$. So there is an element $x_{t_{0}} \in A$ such that $g_{t_{0}}=\widehat{x}_{t_{0}}$. Letting $t_{0}$ vary thorough all the points of $X$ we obtain a function $f$ from $X$ into $A$ defined by

$$
f(t)=x_{t}, \quad t \in X .
$$

We shall next show that $f$ is continuous on $X$. Let $t_{0} \in X, \lambda \in \Lambda$, and $\epsilon>0$ be given. Now $g \in C(X \times \Delta(A))$. So for each $\tau_{0} \in h\left(N_{\lambda}\right)$ there are neighbourhoods $V\left(\tau_{0}\right) \subset \Delta(A)$ of $\tau_{0}$ and $U_{\tau_{0}}\left(t_{0}\right) \subset X$ of $t_{0}$ such that

$$
\left|g(t, \tau)-g\left(t_{0}, \tau_{0}\right)\right|<\frac{\epsilon}{4}, \quad t \in U_{\tau_{0}}\left(t_{0}\right), \quad \tau \in V\left(\tau_{0}\right) .
$$

The sets $\left\{V\left(\tau_{0}\right) \mid \tau_{0} \in h\left(N_{\lambda}\right)\right\}$ form an open cover of the compact set $h\left(N_{\lambda}\right)$. Thus there is a finite subcover of it, say $V_{1}\left(\tau_{1}\right), V_{2}\left(\tau_{2}\right), \ldots, V_{n}\left(\tau_{n}\right)$. Denote by $U_{1}, U_{2}, \ldots, U_{n}$ the corresponding neighbourhoods of $t_{0}$ for which (5) is valid. Let us choose $U\left(t_{0}\right)=\cap_{i=1}^{n} U_{i}$. Now, if $\tau \in h\left(N_{\lambda}\right)$ and $t \in U\left(t_{0}\right)$ are arbitrary, then $\tau \in V_{i}\left(\tau_{i}\right)$ for some $i=1,2, \ldots, n$. Thus

$$
\left|g(t, \tau)-g\left(t_{0}, \tau_{0}\right)\right| \leq\left|g(t, \tau)-g\left(t_{0}, \tau_{i}\right)\right|+\left|g\left(t_{0}, \tau_{i}\right)-g\left(t_{0}, \tau\right)\right|<\frac{\epsilon}{4}+\frac{\epsilon}{4}=\frac{\epsilon}{2}
$$

for all $t \in U\left(t_{0}\right)$. Therefore, we have

$$
\begin{aligned}
p_{\lambda}\left(f(t)-f\left(t_{0}\right)\right) & =p_{\lambda}\left(x_{t}-x_{t_{0}}\right)=p_{h\left(N_{\lambda}\right)}\left(\widehat{x}_{t}-\widehat{x}_{t_{0}}\right) \\
& =\sup _{\tau \in h\left(N_{\lambda}\right)}\left|\widehat{x}_{t}(\tau)-\widehat{x}_{t_{0}}(\tau)\right|=\sup _{\tau \in h\left(N_{\lambda}\right)}\left|g(t, \tau)-g\left(t_{0}, \tau\right)\right| \leq \frac{\epsilon}{2}<\epsilon
\end{aligned}
$$

for all $t \in U\left(t_{0}\right)$. Thus, for arbitrary $\lambda \in \Lambda$ and $\epsilon>0$ there is a neighbourhood $U\left(t_{0}\right)$ of $t_{0}$ such that $f(t) \in f\left(t_{0}\right)+V_{\lambda}(\epsilon)$ for all $t \in U\left(t_{0}\right)$, from which it follows that $f$ is continuous at $t_{0}$, and we have shown that $f \in C(X, A)$. It is easy to see that $\widehat{f}(t, \tau)=g(t, \tau)$ for all $(t, \tau) \in X \times \Delta(A)$, whence $\widehat{f}=g$, which completes the proof of theorem. 
Theorem 3 is a generalization of Yood's result for commutative $B^{*}$-algebra valued functions. (See [21].) See also Hery's result for $C(S)$-valued functions in [12].

Remark. The involution $g \mapsto g^{*}, g \in C(X \times \Delta(A))$, in $C(X \times \Delta(A))$ is defined as usual by taking the complex conjugate of the function $g$. In other words

$$
g^{*}(t, \tau)=\overline{g(t, \tau)}, \quad g \in C(X \times \Delta(A)),(t, \tau) \in X \times \Delta(A) .
$$

If the hyphotheses of Theorem 3 are valid, then it is easy to see that $\left(f^{*}\right)^{\wedge}=$ $\overline{\hat{f}}, f \in C(X, A)$. Thus we can see that the mapping $f \mapsto \widehat{f}, f \in C(X, A)$, preserves the involution in other words it is a *-isomorphism from $C(X, A)$ onto $C(X \times \Delta(A))$.

Corollary 1. Suppose that the hypotheses of Theorem 3 are valid. Then

(i) $C(X, A)^{\wedge}=C(\Delta(C(X, A)))$.

(ii) $p_{(K, \lambda)}(f)=p_{h\left(N_{(K, \lambda)}\right)}(\widehat{f})$ for all $f \in C(X, A), K \in \mathscr{K}$, and $\lambda \in \Lambda$.

Proof. These results follow from Theorem 3, Lemma 5, and Corollary 10 of [5].

Let $K \in \mathscr{K}$ and $\lambda \in \Lambda$ be given. We shall now consider the factor algebra $C(X, A) / N_{(K, \lambda)}$. The norm on $C(X, A) / N_{(K, \lambda)}$ will be defined by a natural way, whence

$$
\dot{p}_{(K, \lambda)}\left(f+N_{(K, \lambda)}\right)=p_{(K, \lambda)}(f), \quad f+N_{(K, \lambda)} \in C(X, A) / N_{(K, \lambda)} .
$$

The topology on $C(X, A) / N_{(K, \lambda)}$ generated by the norm $\dot{p}_{(K, \lambda)}$ will be denoted by $T\left(\dot{p}_{(K, \lambda)}\right)$.

Corollary 2. Let $K \in \mathscr{K}$ and $\lambda \in \Lambda$ be given. If $(A, T(\mathscr{P}))$ is as in Theorem 3 , then the normed algebra $\left(C(X, A) / N_{(K, \lambda)}, T\left(\dot{p}_{(K, \lambda)}\right)\right)$ is complete. Furthermore, $\left(C(X, A) / N_{(K, \lambda)}, T\left(\dot{p}_{(K, \lambda)}\right)\right)$ is isometrically isomorphic to each of the following Banach algebras: $C\left(h\left(N_{(K, \lambda)}\right)\right), C\left(K \times h\left(N_{\lambda}\right)\right), C\left(K, C\left(h\left(N_{\lambda}\right)\right)\right.$, and $C\left(K, A / N_{\lambda}\right)$ where the norm in each of these algebras has been defined in a natural way, in other words, we have

$$
\begin{aligned}
\dot{p}_{(K, \lambda)}\left(f+N_{(K, \lambda)}\right)= & \sup _{\phi \in h\left(N_{(K, \lambda)}\right)}|\widehat{f}(\phi)|=\sup _{(t, \tau) \in K \times N_{\lambda}}|\widehat{f}(t, \tau)| \\
=\sup _{t \in K} p_{h\left(N_{\lambda}\right)}\left(f(t)^{\wedge}\right) & =\sup _{t \in K} \dot{p}_{\lambda}\left(f(t)+N_{\lambda}\right), \\
& f+N_{(K, \lambda)} \in C(X, A) / N_{(K, \lambda)} .
\end{aligned}
$$

Proof. It is easy to see that the mapping $f+N_{(K, \lambda)} \mapsto \widehat{f}_{\mid K \times h\left(N_{\lambda}\right)}=\widehat{f}$ restricted to $K \times h\left(N_{\lambda}\right), f+N_{(K, \lambda)} \in C(X, A) / N_{(K, \lambda)}$ is an isometrical isomorphism from $C(X, A) / N_{(K, \lambda)}$ onto $C\left(K \times h\left(N_{\lambda}\right)\right)$ such that $\dot{p}_{(K, \lambda)}\left(f+N_{(K, \lambda)}\right)=$ $\sup _{(t, \tau) \in K \times h\left(N_{\lambda}\right)}|\widehat{f}(t, \tau)|, f+N_{(K, \lambda)} \in C(X, A) / N_{(K, \lambda)}$. Since $C(K \times \Delta(A))$ with a sup norm is complete, we can see that $\left(C(X, A) / N_{(K, \lambda)}, T\left(\dot{p}_{(K, \lambda)}\right)\right)$ is complete, too. The rest of the theorem follows from Corollary 10 of [5] and from the well-known results of the commutative $B^{*}$-valued functions defined on the compact Hausdorff space. (See, for example, [3].)

If $S$ is a subset of $C(X, A)$, then we shall denote $\widehat{S}=\{\widehat{f} \in C(X \times \Delta(A)) \mid$ $f \in S\}$. 
Corollary 3. Suppose that $(A, T(\mathscr{P}))$ is as in Theorem 3 , and let $J$ be a closed ideal of $(C(X, A), T(\mathscr{K}, \Lambda))$. Then

$$
h(J)=\left\{\phi_{(t, \tau)} \mid(t, \tau) \in h(\widehat{J})\right\} \quad \text { and } \quad h(\widehat{J})=\left\{(t, \tau) \mid \phi_{(t, \tau)} \in h(J)\right\} .
$$

Corollary 4. Suppose that hypotheses of Theorem 3 are valid. If $E$ is a closed subset of $X$ and $I$ a closed ideal of $(A, T(\mathscr{P}))$, then $J(E, A)^{\wedge}=k(E \times \Delta(A))$ and $C(X, I)^{\wedge}=k(X \times h(I))$.

Proof. This result can be shown similarly as the corresponding result for $B^{*}$ algebra valued functions. (See [3], Theorem 4.1.)

\section{ON CLOSED IDEALS OF $(C(X, A), T(\mathscr{K}, \Lambda))$}

The structure of closed ideals of $C(X, A)$ has been described in many papers under various topological assumptions on $X$ and $A$ (see, for example, [1], [3], [5], [8], [11], [18], [19], [20] ). In most of these cases in those descriptions the points of $X$ and some family of closed ideals of $A$ have been used. In some particular cases it is however possible to use also the points of $\Delta(A)$ and some family of closed ideals of $C(X, A)$ depending on those points.

Let $J$ be a closed ideal of $(C(X, A) ; T(\mathscr{K}, \Lambda))$. We shall say that $J$ is a slice ideal of the first type, if $\widehat{J}=k\left(\{t\} \times E_{t}\right)$ for some point $t \in X$ and closed subset $E_{t} \subset \Delta(A)$. If above $E_{t}=\Delta(A)$, then we say that $J$ is a total slice ideal of the first type. Correspondingly $J$ is a slice ideal of the second type, if $\widehat{J}=k\left(E_{\tau} \times\{\tau\}\right)$ for some $\tau \in \Delta(A)$ and closed subset $E_{\tau} \subset X$. If $E_{\tau}=X$, then we shall say that $J$ is a total slice ideal of the second type.

Directly from the definition of total slice ideal and Corollary 4 we obtain the following result.

Corollary 5. If the hypotheses of Theorem 3 are valid, then each total slice ideal of the first type in $(C(X, A), T(\mathscr{K}, \Lambda))$ is of the form $J(\{t\}, A)$ for some $t \in X$ and each total slice ideal of the second type is of the form $C(X, \operatorname{ker} \tau)$ for some $\tau \in \Delta(A)$.

If $A$ is a commutative $B^{*}$-algebra and $X$ is a compact Hausdorff space and if we equip $C(X, A)$ with the topology of compact convergence, then by [3] (Theorem 4.2) for each closed ideal of $C(X, A)$ there are closed subsets $E_{1} \subset X$ and $E_{2} \subset \Delta(A)$ and families $J_{t}, t \in E_{1}$, and $J_{\tau}, \tau \in E_{2}$, of slice ideals of the first and the second types such that $J=\cap_{t \in E_{1}} J_{t}$ and $J=\cap_{\tau \in E_{2}} J_{\tau}$. Now we can prove the corresponding result for the algebra $(C(X, A), T(\mathscr{K}, \Lambda))$, if the hypotheses of Theorem 3 are valid.

Theorem 4. Let $(A, T(\mathscr{P}))$ be a star algebra for which $\Delta(A)$ is locally equicontinuous and $\widehat{A}=C(\Delta(A))$. If $J$ is a closed proper ideal of the algebra $(C(X, A), T(\mathscr{K}, \Lambda))$, then there are subsets $E_{1} \subset X$ and $E_{2} \subset \Delta(A)$ and families $J_{t}, t \in E_{1}$, and $J_{\tau}, \tau \in E_{2}$, of slice ideals of the first and the second types such that $J=\cap_{t \in E_{1}} J_{t}$ and $J=\cap_{\tau \in E_{2}} J_{\tau}$.

Proof. Let $E_{1}=\{t \in X \mid\{t\} \times \Delta(A) \cap h(\widehat{J}) \neq \varnothing\}$. It is easy to see that $E_{1} \neq \varnothing$. Obviously $\{t\} \times \Delta(A) \cap h(\widehat{J})=\{t\} \times E_{t}$ for each $t \in E_{1}$ where $E_{t}$ is some closed subset of $\Delta(A)$ depending on $t$. If we now choose $\widehat{J}_{t}=k\left(\{t\} \times E_{t}\right), t \in$ $E_{1}$, then $J_{t}$ is a slice ideal of the first type for each $t \in E_{1}$ and it can be shown by the similar method as in [3], Theorem 4.2, that $J=\cap_{t \in E_{1}} J_{t}$. 
Correspondingly, if we choose $E_{2}=\{\tau \in \Delta(A) \mid X \times\{\tau\} \cap h(\widehat{J}) \neq \varnothing\}$, then it is easy to see that $E_{2} \neq \varnothing$ and $X \times\{\tau\} \cap h(\widehat{J})=E_{\tau} \times\{\tau\}$ for each $\tau \in E_{2}$ where $E_{\tau}$ is a closed subset of $X$ for each $\tau \in E_{2}$. Now by choosing $\widehat{J}_{\tau}=k\left(E_{\tau} \times\{\tau\}\right), \tau \in E_{2}$, we can see that each $J_{\tau}, \tau \in E_{2}$, is a slice ideal of the second type and $J=\cap_{\tau \in E_{2}} J_{\tau}$.

Remark. It is easy to see that the above set $E_{1}=\{t \in X \mid I(t) \neq A\}$ where $I(t)=\operatorname{cl}(\{f(t) \mid f \in J\})$. Thus $E_{1}$ is not necessarily closed as it has been shown in [5]. Correspondingly it can been shown that the set $E_{2}$ is not necessarily closed. Some conditions which quarantee that the set $E_{1}$ is closed have been given in [5].

Corollary 6. Suppose that the hypotheses of Theorem 4 are valid. If $J$ is a closed proper ideal of $(C(X, A), T(\mathscr{K}, \Lambda))$, then $J=\cap_{t \in E_{1}} J_{\left(t, k\left(E_{t}\right)\right)}$, where $E_{1}=$ $\{t \in X \mid\{t\} \times \Delta(A) \cap h(\widehat{J}) \neq \varnothing\}$ and $E_{t}=\{\tau \in \Delta(A) \mid(t, \tau) \in\{t\} \times \Delta(A) \cap h(\widehat{J})\}$, $t \in E_{1}$.

Proof. We must only notice that $h\left(\left(J_{\left(t, k\left(E_{t}\right)\right)}\right)^{\wedge}\right)=\{t\} \times E_{t}, t \in E_{1}$.

By Theorem 8 of [5] and Corollary 6 we can obtain the following result:

Corollary 7. Suppose that the hypotheses of Theorem 4 are valid. If $J$ is a closed ideal of $(C(X, A), T(\mathscr{K}, \Lambda))$, then $\operatorname{cl}(\{f(t) \mid f \in J\})=k\left(E_{t}\right)$ for each $t \in E_{1}$ where $E_{1}$ and $E_{t}, t \in E_{1}$, are as in Corollary 6.

Corollary 8. Suppose that the hypotheses of Theorem 4 are valid. Let $J$ be a closed ideal of $(C(X, A), T(\mathscr{K}, \Lambda))$. Furthermore for given $K \in \mathscr{K}$ and $\lambda \in \Lambda$ let $E_{(K, \lambda)}=h(J) \cap h\left(N_{(K, \lambda)}\right)$ and denote by $\mathscr{K}_{0}$ and $\Lambda_{0}$ the subfamilies of $\mathscr{K}$ and $\Lambda$ for which $E_{(K, \lambda)} \neq \varnothing, K \in \mathscr{K}_{0}$, and $\lambda \in \Lambda_{0}$. Then $J=$ $\cap\left\{k\left(E_{(K, \lambda)}\right) \mid K \in \mathscr{K}_{0}, \lambda \in \Lambda_{0}\right\}$.

Proof. By Theorem 2 and Theorem 4.2 of [4] $(C(X, A), T(\mathscr{K}, \Lambda))$ has a property of spectral synthesis. Thus we have

$$
\begin{aligned}
J & =k(h(J))=k\left(\cup\left\{E_{(K, \lambda)} \mid K \in \mathscr{K}, \lambda \in \Lambda\right\}\right) \\
& \left.=k\left(\cup\left\{E_{(K, \lambda)}\right) \mid K \in \mathscr{K}_{0}, \lambda \in \Lambda_{0}\right\}\right)=\cap\left\{k\left(E_{(K, \lambda)}\right) \mid K \in \mathscr{K}_{0}, \lambda \in \Lambda_{0}\right\} .
\end{aligned}
$$

Corollary 9. Suppose that the hypotheses of Theorem 4 are valid. If $E$ is a closed subset of $X$ and $I$ is a closed ideal of $(A, T(\mathscr{P}))$, then

(i) $C(X, I)=\cap_{\lambda \in \Lambda_{0}} C\left(X, k\left(E_{\lambda}\right)\right)$ and

(ii) $J(E, A)=\cap_{K \in \mathscr{H}_{0}} J(E \cap K, A)$

where $E_{\lambda}=h(I) \cap h\left(N_{\lambda}\right), \Lambda_{0}=\left\{\lambda \in \Lambda \mid E_{\lambda} \neq \varnothing\right\}$, and $\mathscr{K}_{0}=\{K \in \mathscr{K} \mid E \cap K$ $\neq \varnothing\}$.

Proof. Part (i) follows from Corollary 4.1 of [4] and Lemma 2 of [6]. For the proof of part (ii) see [3], Theorem 3.3.

In Theorem 4 we sliced the hull of the ideal $\widehat{J}$ by using the points of $X$ and $\Delta(A)$. We can slice $h(\widehat{J})$ also by using certain subsets of $X$ and $\Delta(A)$. So let $J$ be a closed proper ideal of $(C(X, A), T(\mathscr{K}, \Lambda))$. For elements $K \in \mathscr{K}$ and $\lambda \in \Lambda$ denote

$$
\widehat{J}_{K}=k(h(\widehat{J}) \cap K \times \Delta(A))
$$


and

$$
\widehat{J_{\lambda}}=k\left(h(\widehat{J}) \cap X \times h\left(N_{\lambda}\right)\right) .
$$

Theorem 5. Let $(A, T(\mathscr{P}))$ be a star algebra for which $\Delta(A)$ is locally equicontinuous and $\hat{A}=C(\Delta(A))$. If $J$ is a closed ideal of $(C(X, A), T(\mathscr{K}, \Lambda))$, then

$$
J=\cap_{K \in \mathscr{K}_{1}} J_{K} \quad \text { and } \quad J=\cap_{\lambda \in \Lambda_{1}} J_{\lambda}
$$

where $\widehat{J}_{K}, K \in \mathscr{K}_{1}$, and $\widehat{J}_{\lambda}, \lambda \in \Lambda_{1}$, are ideals defined in (6) and (7) and $\mathscr{K}_{1}=\{K \in \mathscr{K} \mid h(\widehat{J}) \cap K \times \Delta(A) \neq \varnothing\}$ and $\Lambda_{1}=\left\{\lambda \in \Lambda \mid h(\widehat{J}) \cap X \times h\left(N_{\lambda}\right) \neq \varnothing\right\}$. Proof. It is easy to see that for any $K \in \mathscr{K}$ and $\lambda \in \Lambda$ we have $J_{K}=\cap_{t \in K} J_{t}$ and $J_{\lambda}=\cap_{\tau \in h\left(N_{\lambda}\right)} J_{\tau}$ where the ideals $J_{t}, t \in K$, and $J_{\tau}, \tau \in h\left(N_{\lambda}\right)$, are ideals defined in the proof of the theorem 4. Now the results of the theorem follow directly from Theorem 4.

Next we shall consider the case in which $(C(X, A), T(\mathscr{K}, \Lambda))$ is normal. We recall that a topological algebra $(A, T(\mathscr{P}))$ is normal, if the elements of $\hat{A}$ separate any two disjoint closed subsets $F_{1}$ and $F_{2}$ of the carrier space $\Delta(A)$. If $X$ is a normal topological space and $(A, T(\mathscr{P}))$ is a normal topological algebra, then $(C(X, A), T(\mathscr{K}, \Lambda))$ is not necessarily normal. Namely, the product space $X \times \Delta(A)$ is not necessarily normal even if $X$ and $\Delta(A)$ both would be normal spaces. (See [10], p. 144.) However, if $(C(X, A), T(\mathscr{K}, \Lambda))$ is a normal topological algebra, then both $X$ and $(A, T(\mathscr{P}))$ must be normal.

Theorem 6. Let $(A, T(\mathscr{P}))$ be a star algebra for which $\Delta(A)$ is locally equicontinuous and $\widehat{A}=C(\Delta(A))$. Furthermore suppose that $(C(X, A), T(\mathscr{K}, \Lambda))$ is a normal topological algebra. If $J$ is a closed proper ideal of $(C(X, A), T(\mathscr{K}, \Lambda))$, then:

(i) $J=\cap\left\{J+N_{(K, \lambda)} \mid K \in \mathscr{K}_{0}, \lambda \in \Lambda_{0}\right\}$ where $\mathscr{K}_{0}$ and $\Lambda_{0}$ are subfamilies of $\mathscr{K}$ and $\Lambda$ for which $h(\widehat{J}) \cap K \times h\left(N_{\lambda}\right) \neq \varnothing$.

(ii) $J=\cap\left\{J+J(\{t\}, A) \mid t \in E_{1}\right\}$ where $E_{1}=\{t \in X \mid\{t\} \times \Delta(A) \cap h(\widehat{J})$ $\neq \varnothing\}$.

(iii) $J=\cap\left\{J+C(X, \operatorname{ker} \tau) \mid \tau \in E_{2}\right\}$ where $E_{2}=\{\tau \in \Delta(A) \mid X \times\{\tau\} \cap$ $h(\widehat{J}) \neq \varnothing\}$.

(iv) $J=\cap\left\{J+C\left(X, N_{\lambda}\right) \mid \lambda \in \Lambda_{1}\right\}$ where $\Lambda_{1}=\left\{\lambda \in \Lambda \mid X \times h\left(N_{\lambda}\right) \cap h(\widehat{J})\right.$ $\neq \varnothing\}$.

(v) $J=\cap\left\{J+J(K, A) \mid K \in \mathscr{K}_{1}\right\}$ where $\mathscr{K}_{1}=\{K \in \mathscr{K} \mid K \times \Delta(A) \cap h(\widehat{J})$ $\neq \varnothing\}$.

Proof. By Corollary 4.2 of [5] for each closed ideals $I_{1}$ and $I_{2}$ of the normal star algebra $(A, T(\mathscr{P}))$ for which $\hat{A}=C(\Delta(A))$, the sum ideal $I_{1}+I_{2}$ is either the closed ideal of $(A, T(\mathscr{P}))$ or otherwise $I_{1}+I_{2}=A$. Now by using this result and Theorem 3.3 of [4] it is easy to see that (i) $-(v)$ of the theorem are valid.

Remark. The ideals $J+J(\{t\}, A), t \in E_{1}$, of Theorem 5 are just the ideals $J_{t}, t \in E_{1}$, of Theorem 4. Namely, we have

$$
\left(J+J(\{t\}, A)^{\wedge}=k\left(h\left(\widehat{J}+J(\{t\}, A)^{\curlywedge}\right)=k(h(\widehat{J}) \cap\{t\} \times \Delta(A))=\widehat{J}_{t}\right.\right.
$$


for each $t \in E_{1}$ and thus $J+J(\{t\}, A)=J_{t}, t \in E_{1}$. Correspondingly we can show that

$$
(J+C(X, \operatorname{ker} \tau))^{\wedge}=\widehat{J}_{\tau}, \quad \tau \in E_{2},
$$

and therefore, we have $J+C(X, \operatorname{ker} \tau)=J_{\tau}$ for each $\tau \in E_{2}$ where the ideals $J_{\tau}$ are as in Theorem 4. Futhermore, it is easy to see that $J+J(K, A)=$ $J_{K}, K \in \mathscr{K}_{1}$, and $J+C\left(X, N_{\lambda}\right)=J_{\lambda}, \lambda \in \Lambda_{1}$, where $J_{K}$ and $J_{\lambda}$ are ideals defined in (6) and (7).

\section{REFERENCES}

1. M. Abel, Description of closed ideals in algebras of continuous vector-valued functions, Math. Notes, vol. 30, Princeton Univ. Press, Princeton, NJ, 1981, pp. 887-892.

2. R. Arens, A generalization of normed rings, Pacific J. Math. 4 (1952), 455-471.

3. J. Arhippainen, On the ideal structure and approximation properties of algebras of continuous $B^{*}$-algebra valued functions, Acta Univ. Oulu. Ser. A Sci. Rerum Natur. 187 (1987).

4. $ـ$ On commutative locally m-convex algebras, Acta et Comm. Univ. Tartuensis 928 (1991), 15-28.

5. _ On the ideal structure of algebras of LMC-algebra valued functions, Studia Math. 101 (1992), 311-318.

6. __ On locally convex square algebras, Preprint series in Math., Univ. of Oulu, 1992.

7. E. Beckenstein, L. Narici, and S. Suffel, Topological algebras, North-Holland, New York, 1977.

8. W. Dietrich, The maximal ideal space of the topological algebra $C(X, E)$, Math. Ann. 183 (1969), 201-212.

9. __ Function algebras on completely regular spaces, Diss., Northwestern Univ., Evanston, IL, 1971.

10. J. Dugundji, Topology, Allyn and Bacon, Boston, 1966.

11. W. Hery, Rings of continuous Banach algebra-valued functions, Doct. Diss. Absttrs 45, Polytech. Instit. of New York, 1974.

12. - Maximal ideals in algebras of continuous $C(S)$ valued functions, Atti Acad. Naz. Lincei Rend. Cl. Sci. Fis. Mat. Natur. (8) 58 (1975), 195-199.

13. __ Maximal ideals in algebras of topological algebra valued functions, Pacific J. Math. 65 (1976), 365-373.

14. A. Mallios, Topological algebras selected topics, Elsevier, New York, 1986.

15. E. Michael, Locally multiplicatively m-convex topological algebras, Mem. Amer. Math. Soc., vol. 11, Amer. Math. Soc., Providence, RI, 1952.

16. P. Morris and D. Wulbert, Functional representation of topological algebras, Pacific J. Math. 22 (1967), 323-337.

17. L. Nachbin, Elements of approximation theory, Van Nostrand, Princeton, NJ, 1967.

18. J. Prolla, Approximation of vector-valued functions, North-Holland, Amsterdam, 1977.

19. __ On the spectra of non-Archimedean function algebras, Lecture Notes in Math., vol. 843, Springer-Verlag, New York, 1980, pp. 547-560.

20. __ Topological algebras of vector-valued continuous functions, Math. Anal. Appl. Part B. Adv. in Math. Supplementary Studies. Vol. B (1981), 727-740.

21. B. Yood, Banach algebras of continuous functions, Pacific J. Math. 3 (1951), 30-48.

Department of Mathematics, University of Oulu, SF- 90570 Oulu, Finland

E-mail address: mathdept@finou. oulu.fi 